\title{
Optimization of actuating elements of transport control systems with magnetic levitation based on the solution of inverse problems
}

\author{
Anna Balaban ${ }^{1}$, Yuri Bakhvalov ${ }^{1}$, and Valery Grechikhin ${ }^{2, *}$ \\ ${ }^{1}$ Department of Applied Mathematics, SRSPU (NPI), 346428 Novocherkassk, Russia \\ ${ }^{2}$ Department of Information and Measuring Systems and Technologies, SRSPU (NPI), 346428 \\ Novocherkassk, Russia
}

\begin{abstract}
The designing of optimal energy-saving actuating elements for transport control systems with magnetic levitation (electromagnets with current windings and permanent magnets) is reduced to solving the following inverse problem. It is necessary to determine the dimensions and magnetomotive force of the actuating element, providing a given levitation force, magnetic induction in ferromagnets and a minimum mass of the actuating element. The solution of the direct problem (determination of the force of levitation and magnetic induction) is carried out by the finite element method. In the problem, the objective function is the mass of the actuating element and the restrictions are superimposed on the force and magnetic induction. Solving this optimization problem by the Lagrange method leads to inefficient algorithm. The article describes the proposed optimization method based on the transformation of constraints into objective functions, that is, the reduction of the problem to the multicriteria problem with consistent minimization of objective functions with a limited number of required parameters. A number of criteria are minimized analytically. The application of the complex criterion is considered. To reduce the time of solving the problem, the hierarchy of models is used: chain and field model. The proposed optimization method allows reducing the time of solving the problem by about two times. The cost of electricity due to the use of permanent magnets is reduced by four times. An example of optimization of the design of the actuating element of transport control system with magnetic levitation is considered.
\end{abstract}

\section{Introduction}

Transport plays an important role in modern society. In this regard, many countries are searching for environmentally friendly, noiseless, economical modes of transport. One of the directions of the search is the replacement of the railway transport hub "wheel-rail" contactless magnetic suspension. Such transport provides for the use of a linear traction electric drive and is capable of speeds up to $500 \mathrm{~km} / \mathrm{h}$ [1]. In China and Japan, commercial transport systems with magnetic levitation of crews are successfully operated. Magnetic

* Corresponding author: vgrech@mail.ru 
suspension is increasingly used in instrument making and mechanical engineering (magnetic bearings, positioning systems, etc.). In connection with the above, the actual problem is the development of effective methods for optimizing the actuating elements (AE) of levitation and lateral stabilization systems.

Further, the research methodology based on solving inverse problems is used, which is one of the new directions in the design, optimization and identification of technical objects [1]. As is known, inverse problems refer to ill-posed ones in the classical sense. The nonuniqueness and instability of their solutions are possible. In the article only conditionally correct (correct according by A. N. Tikhonov) problems are considered. This means that the existence and uniqueness of the solutions of these problems are proved [26]. To ensure stability, the class for finding solutions is narrowed, that is, the sought solution is searched in class of bounded functions with the norm $\|\varphi\|<N$, where $\mathrm{N}-$ positive constant [7].

\section{Optimization method}

$\mathrm{AE}$ are electromagnets containing current windings and permanent magnets (PM). PM are used to save electricity.

$\mathrm{AE}$ are installed on an arm connected to a vehicle (Fig. 1, where is denoted: (1) support; (2-5) ferrorail of the levitation system, core of the levitation electromagnet, PM system of levitation, coil; (6-9) coil, PM, core of the lateral-stabilization electromagnet, ferrorail of the lateral-stabilization system; (10) arm).

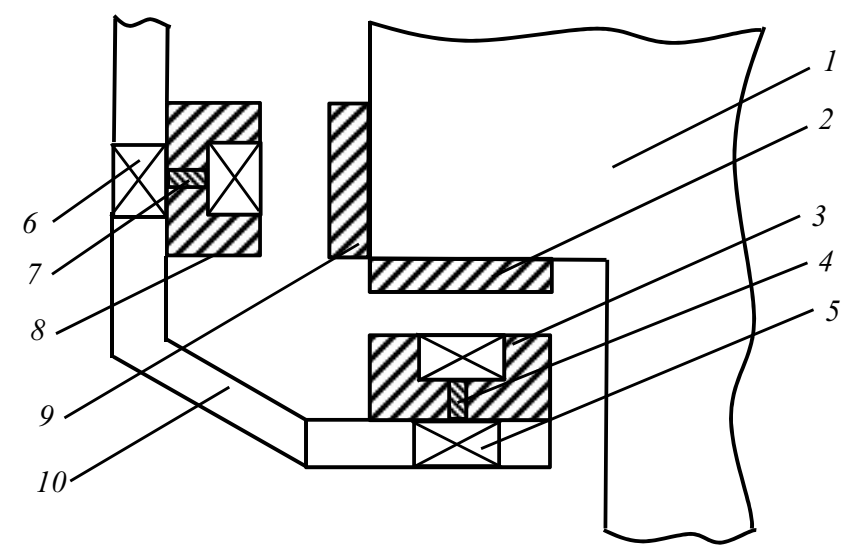

Fig. 1. The location of the actuating elements on an arm connected to a vehicle.

The designations of the dimensions and the construction of the AE further examined are shown in Fig. 2. The designations of the dimensions and the construction of the AE examined below are shown in Fig. 2. Since the constructions of the AE levitation and lateral stabilization are similar, therefore, consider the design of the AE optimal levitation.

When optimizing the AE, the hierarchy of mathematical models is used for reduction of the total time of the problem solution. $\mathrm{AE}$ is represented by a magnetic circuit in the first stage (Fig. 3). In the figure: $R_{s t}, R_{g}, R_{c}$ - magnetic resistance of ferro-rail, gap and core; $i w$ - magnetomotive force of the coil; $M$ - the magnetization of PM. 


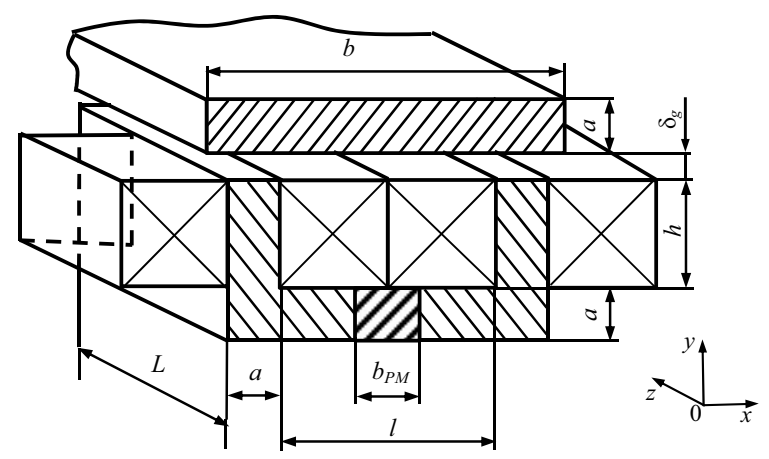

Fig. 2. The design of the actuating element. Designation of dimensions.

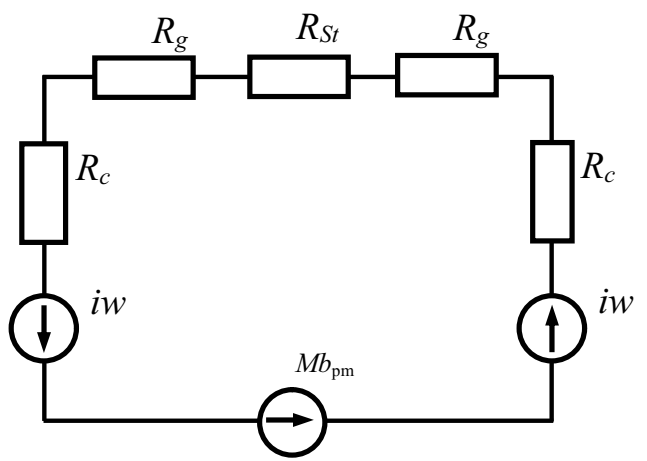

Fig. 3. Equivalent replacement circuit of the actuating element.

The initial data: $B_{\delta}$ - maximum magnetic induction in the gap, $L$ - length of AE along the $0 z$ axis, permissible errors $\delta_{F}$ and $\delta_{B}$ in the definition $F_{l}$ and $B_{\delta}$ accordingly, $M_{c}-$ crew mass, $n$ - number of AE of crew, non-magnetic gap $\delta_{g}$.

The required levitation force $F_{l}$ of each $\mathrm{AE}$ is determined by the formula

$$
F_{l}=M_{c} g / n
$$

where $g$ is gravitational acceleration.

The maximum magnetic induction is taken to be $0,5 \mathrm{~T}$, which provides an unsaturated condition of ferromagnets and an effective work of the gap regulator. The value of $L$ is taken equal to $1 \mathrm{~m}$, and it is established that a plane-parallel approximation can be used when modeling the magnetic field. Values of errors $\delta_{F}=\delta_{B}=0,01$.

It is required to determine the geometric dimensions of $\mathrm{AE}: b, b_{P M}, l, h, a$; magnetomotive force of the coil $i w$, and also to minimize the mass of AE with the following restrictions:

$$
\left.\begin{array}{c}
F_{l}<F_{l}^{(k)} \leq F_{l}\left(1+\delta_{F}\right) ; B_{\delta} \leq B_{\delta}^{(k)} \leq B_{\delta}\left(1+\delta_{B}\right) \\
\left|b^{(k+1)}-b^{(k)}\right| \leq \delta b^{(k+1)} ;\left|b_{P M}^{(k+1)}-b_{P M}^{(k)}\right| \leq \delta b_{P M}^{(k+1)} ;\left|l^{(k+1)}-l^{(k)}\right| \leq \delta l^{(k+1)} ; \\
\left|h^{(k+1)}-h^{(k)}\right| \leq \delta h^{(k+1)} ;\left|a^{(k+1)}-a^{(k)}\right| \leq \delta a^{(k+1)} ;\left|i w^{(k+1)}-i w^{(k)}\right| \leq \delta i w^{(k+1)},
\end{array}\right\}
$$


where $\delta=0,01$. In formulas (2), (3), the superscript means that the value considered is found at the $k$-th step of the algorithm.

The initial values of the unknown quantities are found by means of a magnetic circuit, solving analytically the inverse problem.

At the second stage, a more rigorous field model is used to refine the unknown quantities.

The value $b_{P M}^{(0)}$ is determined using the first model. Let the magnetic permeability of ferromagnets be infinite. On the basis of relation

$$
\oint_{l} \vec{H} d \vec{l}=i w
$$

when $i w=0$ is obtained

$$
H_{\delta} 2 \delta_{g}+H_{P M} b_{P M}=0
$$

where $H_{\delta}=B_{\delta}^{*} / \mu_{0} ; \quad H_{P M}=B_{\delta}^{*} / \mu_{0}-M$, where $\mu_{0}-$ magnetic constant, $\mu_{0}=4 \pi \cdot 10^{-7} \mathrm{GN} / \mathrm{m} ; M-$ magnetization of PM, known for the selected brand of PM. Let us assume that PM creates $B_{\delta}^{*}=0,5 B_{\delta}$. Then

$$
b_{P M}^{(0)}=\frac{B_{\delta} \delta_{g} / \mu_{0}}{M-0,5 B_{\delta} / \mu_{0}} .
$$

The value $b_{P M}^{(0)}$ is clarified at the end of the second stage.

Let us assume that magnetomotive force of the coil creates $B_{\delta}^{*}=0,5 B_{\delta}$ and $M=0$. Then, on the basis of (4) is obtained

$$
i w^{(0)}=\frac{0,5 B_{\delta}}{\mu_{0}}=\frac{B_{\delta} \delta}{4 \mu_{0}}\left(2 \delta_{g}+b_{P M}^{(0)}\right) .
$$

The square of the coil aperture is determine by the equation

$$
S_{c}^{(0)}=l^{(0)} h^{(0)}=\frac{2 i w^{(0)}}{k_{f} j},
$$

where $k_{f}$ - is the aperture occupation ratio in respect to copper, which is taken equal to 0.7 ; $j$ - current density in a coil, $j=3 \cdot 10^{6} \mathrm{~A} / \mathrm{m}^{2}$.

As follows from Eq. (7),

$$
l^{(0)}=\frac{S_{c}^{(0)}}{h^{(0)}}=\frac{2 i w^{(0)}}{k_{f} j h^{(0)}} .
$$

The levitation force of the $\mathrm{AE}$ is determined by the following approximate formula 


$$
F_{l}=2 \frac{B_{\delta}^{2} S_{p} k_{b}}{2 \mu_{0}},
$$

where $S_{p}$ - pole square; $k_{b}$ - is a factor taking into account buckling of the magniflux, $k_{b}=1,2 \div 1,5$.

The pole square is determined from formula

$$
S_{p}=a L
$$

On the basis of (9) and (10) is received

$$
a^{(0}=\frac{S_{p}^{(0)}}{L}=\frac{\mu_{0} F_{l}}{B_{\delta}^{2} k_{b} L} .
$$

The AE mass (objective function) is determined by the equation

$$
M_{e m}=\left[2 a h+\left(\frac{S_{c}}{h}+2 a\right) a\right] L \rho_{S t}+2 S_{c} k_{f} L \rho_{\mathrm{Cu}},
$$

where $\rho_{S t}, \rho_{C u}$ are the densities of ferromagnetic (steel) and copper accordingly.

The unknown quantity in (11) is $h$. The value of $h$ at which $M_{e m}$ is minimal is determined by the formula

$$
h^{(0)}=\sqrt{\frac{S_{c}^{(0)}}{2}}=\sqrt{\frac{i w^{(0)}}{k_{f} j}} .
$$

The width of the ferro-rail is determined by the formula

$$
b^{(0)}=l^{(0)}+4 a^{(0)} .
$$

After determining the initial values of the required parameters, it is necessary to clarify them by applying the field model of the problem. In this case, it is necessary to solve the optimization problem with the objective function (mass) $M_{e m}$, constraints (2)-(4) and initial values of the required parameters. Such a problem can be solved by the Lagrange method. However, in practice this method is ineffective, since it leads to the solution of a nonlinear system of equations of large dimension.

Consider the application of the developed optimization method based on the transformation of constraints to objective functions and the consequent minimization of the obtained criteria for a limited number of variables [8, 9].

On the basis of relations (2) and (11), the following system of criteria is constructed

$$
J_{1}\left(S_{p}\right)=\left(F_{l}^{(k)}-\left(1+\delta_{F}\right) F_{l}\right)^{2} ; J_{2}(i w)=\left(B_{\delta}^{(k)}-\left(1+\delta_{B}\right) B_{\delta}\right)^{2} ; J_{3}(h)=M_{e m} .
$$

Further, using the algorithm for solving the inverse problem [9], the values of the required parameters are clarified. 


\section{Results of computational experiment}

Let's consider an example. It is needed to determine the dimensions and magnetomotive force of electromagnets with a minimum mass of the crew levitation system of mass $M=60 \mathrm{t}$, where $n=20$ of AE.

Assume, that $j=3 \cdot 10^{6} \mathrm{~A} / \mathrm{m}^{2} ; \quad B_{\delta}=0.7 \mathrm{~T} ; \quad k_{b}=1.5 ; \quad k_{z}=0.7 ; \quad \delta=0.015 \mathrm{~m}$; $\varepsilon_{1}\left(B_{\delta}^{*}\right)=0.01 ; \varepsilon\left(F_{l}\right)=0.01$.

The initial values of the unknown parameters are determined using the formulas (1), (6)(10), (12), (13): $F_{l}=30 \quad \mathrm{hN} ; \quad a^{(0)}=0.025 \mathrm{~m} ; \quad i w^{(0)}=6768 \mathrm{~A} ; \quad h^{(0)}=0.052 \mathrm{~m}$; $l^{(0)}=0.103 \mathrm{~m} ; b^{(0)}=0.17 \mathrm{~m} ; b_{P M}^{(0)}=0.0037 \mathrm{~m}$.

In the second stage, at the seventh iteration is obtained: $a^{(7)}=0.0357 \mathrm{~m} ; i w^{(7)}=16920 \mathrm{~A}$; $h^{(7)}=0.053 \mathrm{~m} ; l^{(7)}=0.106 \mathrm{~m} ; b^{(7)}=0.197 \mathrm{~m} ; b_{P M}^{(7)}=0.004 \mathrm{~m}$.

\section{Conclusion}

The article describes the proposed optimization method based on the transformation of constraints into objective functions, that is, the reduction of the problem to the multicriteria problem with consistent minimization of objective functions with a limited number of required parameters. One of the objective functions is minimized analytically. The method allows to reduce the time of solving the problem by approximately two times. The design of optimal devices is based on the solution of conditionally correct inverse problems. The cost of electricity due to the use of permanent magnets is reduced by four times. The method can be used for the design and identification of various electrical devices.

The reported study was funded by RFBR according to the research project \#18-01-00204\18 «Improvement of methods solving the inverse problems for identification magnetization of permanent magnets in electrical devices using the additional regularizing information».

\section{References}

1. Y.A. Bakhvalov, N.I. Gorbatenko, V.V. Grechikhin, A.L. Yufanova, Russian Electrical Engineering, 88, 1, 15 (2017)

2. O.M. Alifanov, Inverse heat transfer problems (Springer-Verlag, NY, 1994)

3. V.T. Borukhov, I.V.Gaishun, V.I. Timoshpol'skii, Structural properties of dynamic systems and inverse problems of mathematical physics (Beloruskaya nauka, Minsk, 2009)

4. A.O. Vatulyan, Inverse Problems in Solid Mechanics (Phismatlit, Moscow, 2007)

5. N.V. Korovkin, V.L. Chechurin, M. Hayakawa, Inverse Problems in Electric Circuits and Electromagnetics (Springer, NY, 2006)

6. A.N. Tikhonov, V.Y. Arsenin, Solution of Ill-Posed Problems (Winston \& Sons, Washington, 1977)

7. A.A. Samarsky, L.P. Vabishchevich, Numerical methods for solving inverse problems of mathematical physics (LKI Publ, Moskow, 2009)

8. A.L. Balaban, Russian Electromechanics, 4, 34 (2017)

9. A.L. Balaban, Yu.A. Bakhvalov, V.V. Grechikhin, D.V. Shaykhutdinov JEAS, 13, 7, $1696(2018)$ 